\title{
Life after Autologous Hematopoietic Stem Cell Transplantation for Systemic Sclerosis
}

\author{
Daniela A Moraes' \\ Maria Carolina Oliveira (iD) ${ }^{2}$ \\ 'Division of Clinical Immunology, \\ Department of Internal Medicine, \\ Ribeirão Preto Medical School, University \\ of São Paulo, Ribeirão Preto, Brazil; \\ ${ }^{2}$ Center for Cell-Based Therapy, Ribeirão \\ Preto Medical School, University of São \\ Paulo, Ribeirão Preto, Brazil
}

\begin{abstract}
Stem cell transplantation has been investigated as treatment for severe and progressive systemic sclerosis (SSc) for the past 25 years. To date, more than $1000 \mathrm{SSc}$ patients have been transplanted worldwide. Overall and event-free survival have increased over the years, reflecting stricter patient selection criteria and better clinical management strategies. This review addresses long-term outcomes of transplanted SSc patients, considering phase I/II and randomized clinical trials, as well as observational studies and those assessing specific aspects of the disease. Clinical outcomes are discussed comparatively between studies, highlighting advances, drawbacks and controversies in the field. Areas for future development are also discussed.
\end{abstract}

Keywords: systemic sclerosis, stem cell transplantation, long-term outcomes, progressionfree survival

\section{Introduction}

Systemic sclerosis (SSc) is a chronic autoimmune disease characterized by tissue fibrosis, pronounced alterations in the microvasculature and frequent abnormalities in cellular and humoral immunity. ${ }^{1}$ Combined pathogenic mechanisms of inflammation, fibrosis and microvascular damage affect the skin and internal organs, including the lungs, heart, gastrointestinal tract and kidneys. ${ }^{2}$ Conventional treatment includes systemic immunosuppression, vasodilators and more recently, antifibrotic therapy. ${ }^{3}$ However, a subset of patients with severe and progressive disease is refractory to these approaches. A meta-analysis from 2012 showed that despite newly available medications, more standardized treatment protocols and strategies to enable early diagnosis, mortality in SSc had not decreased in 40 years. ${ }^{4}$ In fact, none of the available conventional treatments reverse the natural course of the disease or demonstrate prolonged benefit. ${ }^{5}$ Currently, interstitial lung disease, pulmonary hypertension and cardiac involvement are the major causes of death in patients with SSc. ${ }^{2,6}$ Patients with rapidly progressive cutaneous involvement and visceral involvement have poor prognosis, with mortality rates reaching $30 \%$ after 5 years of diagnosis, despite conventional treatment. ${ }^{7,8}$

In the mid-1990s, given the lack of effective therapeutic options for refractory autoimmune diseases, and after reports of patients who underwent stem cell transplantation for hematological indications but that presented improvement of coincidental autoimmune conditions, autologous hematopoietic stem cell transplantation (AHSCT) was considered as treatment for patients with severe SSc. ${ }^{9,10}$ Since then, several studies with series of patients and phase I/II clinical trials have shown the
Correspondence: Maria Carolina Oliveira Department of Internal Medicine,

Ribeirão Preto Medical School, University of São Paulo, Avenida dos Bandeirantes, 3900, Ribeirão Preto, SP, I4048-900,

Brazil

Email mcarolor@usp.br 
efficacy of AHSCT in SSc. ${ }^{11-15}$ More recently, randomized controlled trials have demonstrated the superiority of transplantation over conventional treatment. ${ }^{16-18}$

Experience with AHSCT for SSc has increased over time, and protocols have been refined, in special regarding patient selection. Consensus meetings and discussions within medical specialty societies also established recommendations and guidelines to improve patient outcomes. $^{9,19-21}$ Today, almost 25 years after the first transplant, SSc is the most frequently transplanted rheumatic disease in the world. Toxicity associated with the procedure has decreased and long-term disease control has improved. As a consequence, transplanted SSc patients have lived longer lives and it is possible to collect data from long-term follow-up. In this study, we revisit the literature, with special emphasis on the last 10 years, discussing short and long-term clinical outcomes of patients with SSc undergoing AHSCT.

We searched the literature in PubMed and Science Direct databases, within a defined period from 1995 to 2021, using words "stem cell transplantation" and "systemic sclerosis". Only studies in English were included. The articles were initially evaluated by title and abstract and, if necessary, in more detail. Among the available articles, only those with clinical data on autologous hematopoietic stem cell transplantation were selected. Research articles were preferred over case reports, review articles, commentaries, and editorials. Studies were excluded if included less than 5 patients, addressed conditions different than systemic sclerosis or included only pediatric patients. We mostly selected articles from the past 10 years, although older important publications have been referenced. Within the selected articles, the following data were extracted: transplant-related mortality, overall survival, progression-free survival, relapse or progression of SSc, disease progression, changes in modified Rodnan Skin Score (mRSS), changes in lung function, quality of life, fertility and long-term complications such as malignancies and secondary autoimmune diseases.

\section{Transplant Procedure}

Autologous stem cell transplantation is a form of intensive immunotherapy that aims to eradicate the autoreactive adaptive immune system. Autologous stem cells are harvested and cryopreserved before beginning of the procedure. These cells, thawed and reinfused intravenously to the patient after administration of an immunoablative conditioning regimen, provide accelerated hematopoietic reconstitution and enables reinstatement of a renewed immune system, with long-lasting tolerance to autoantigens (Figure 1). Autologous hematopoietic stem cell transplantation deeply modifies the immune system, promoting an immunological balance that halts inflammation and tissue destruction, enabling disease control, and, to some degree, tissue repair. ${ }^{22}$

Most transplant centers use a non-myeloablative conditioning regimen consisting of high doses of cyclophosphamide plus anti-thymocyte globulin. Higher intensity regimens including total body irradiation or thiotepa have been preferred by a few centers. ${ }^{14,18,23,24}$ Different regimens have been compared and discussed for their advantages and drawbacks, mainly addressing aspects related to safety and efficacy, but so far, there are no specific recommendations. $^{25}$ There is also considerable debate about the benefits of graft selection, as numerous transplant centers manipulate the harvested autologous hematopoietic stem cells before cryopreservation, positively selecting the graft for CD34+ cells. Graft selection eliminates most mature lymphocytes and may reduce the risks of reinfusing autoreactive cells within the graft. On the

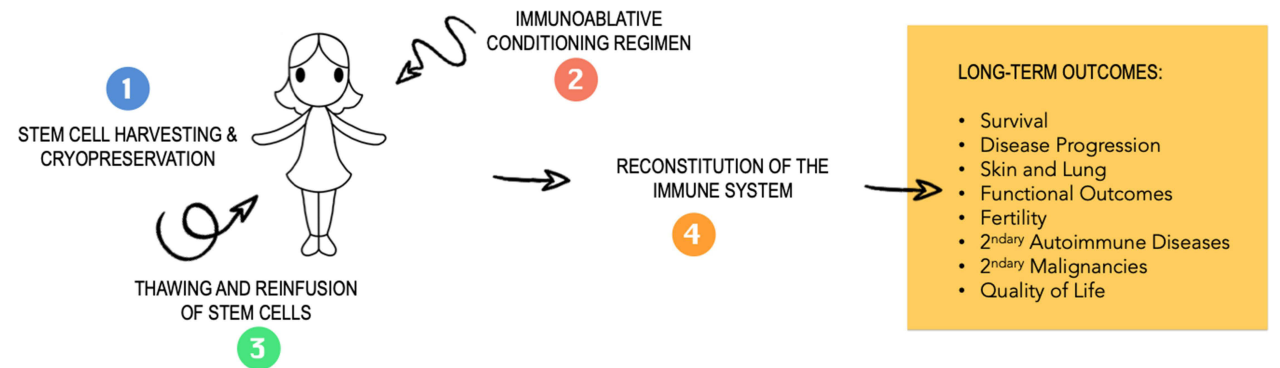

Figure I Schematic representation of the transplant procedure. Autologous stem cells are mobilized from the bone marrow to the peripheral blood, from where they are harvested by leukapheresis and cryopreserved (I). The graft can be selected before cryopreservation or remain unmanipulated. The patient then undergoes an immunoablative conditioning regimen (2), followed by intravenous administration of the autologous cells, which are thawed immediately before infusion (3). After a period of aplasia, there is reconstitution of the immune system (4), and the patient is discharged from the hospital. Long-term outcomes are evaluated over time. 
other hand, it delays immunological recovery and may increase the incidence of viral infections after AHSCT. $^{26,27}$ Attempts to compare clinical outcomes from patients transplanted with or without graft selection have conflicting results, and further prospective investigations are needed. ${ }^{28-30}$

\section{Overall Survival and Toxicity}

Since the initial reports from the end of the last century, numerous phase I/II studies have shown feasibility and beneficial outcomes of AHSCT (Table 1). ${ }^{1-15}$ In the last decade, three randomized controlled studies - ASSIST (Autologous Systemic Sclerosis Immune Suppression Trial, 2011), ASTIS (Autologous Stem Cell Transplantation versus Immunosuppression trial, 2014) and SCOT (Scleroderma: Cyclophosphamide or Transplantation trial, 2018) - have shown that AHSCT is superior in efficacy and safety than monthly intravenous cyclophosphamide (CY) pulses (Table 2) ${ }^{16-18} \mathrm{~A}$ fourth study retrospectively compared the results of AHSCT with those of a historical cohort of SSc patients who shared similar clinical characteristics. ${ }^{38}$ This was a singlecenter study, and updated knowledge on patient selection and intra-transplant management translated into lower transplant-related mortality and higher overall survival. Table 2 describes the main characteristics and patient outcomes from each trial.

ASSIST was a groundbreaking study in 2011, and the first to show benefits of transplantation over conventional therapy, despite the small number of enrolled patients and short follow-up of only 2 years. ${ }^{16}$ According to the authors, study enrollment was stopped earlier than originally planned. An interim analysis showed failure of equipoise, since there was significant difference in outcomes between groups after inclusion of only 19 patients, favoring transplant. ASTIS, published in 2014, was a multicenter European trial that had a higher transplantrelated mortality and lower overall survival than the other two studies. ${ }^{17}$ The higher transplant-related toxicity of ASTIS was ascribed to the lack of a thorough evaluation of patients for cardiac involvement before transplantation. ${ }^{39}$ The importance of cardiac screening only became fully known and incorporated into practice around 2010, at the end of patient recruitment in ASTIS. $^{32,40}$ As a result, it is possible that patients with severe cardiac involvement were enrolled for transplantation, increasing the death rate. Nevertheless, after 1 year of follow-up, in accordance with the other comparative trials,
ASTIS showed superiority of transplantation over conventional cyclophosphamide treatment.

The SCOT trial used a myeloablative and thus highintensity conditioning regimen, including total body irradiation. ${ }^{18}$ Although the described transplant-related mortality was acceptable in this trial, and lower than that of ASTIS, the $85 \%$ incidence of major (grade 4) transplant-related adverse effects evidences the potential toxicity of the regimen. In the past, total body irradiation, which included irradiation of the lung tissue, was associated with severe adverse events and high mortality in transplants for SSc patients. ${ }^{11}$ Total body irradiation regimens may also be associated with scleroderma renal crises, as patients undergoing AHSCT that include TBI are more likely to develop acute kidney insufficiency. ${ }^{41}$ As a result of this experience, lung and renal shielding are adopted in TBI-based regimens for SSc patients. ${ }^{18,42}$ The available evidence in the literature is not sufficient to define whether the intensity of the transplant regimen associates with better or worse clinical outcomes. Higher intensity regimens may provide more efficient and longlasting eradication of the autoreactive immune system, but non-myeloablative regimens may be safer. It is most likely that multiple factors are involved and that other aspects, such as patient selection, have a stronger influence on the process.

Transplant-associated cardiac toxicity is a current concern in AHSCT for SSc patients. Most conditioning regimens include high doses of cyclophosphamide, and this alkylating agent is associated with dose-dependent acute myocardial injury through direct endothelial capillary damage. ${ }^{43}$ On the other hand, cardiac involvement is a frequent and underdiagnosed manifestation of SSc, as patients may be asymptomatic, and at early stages, echocardiography may overlook diastolic dysfunction. ${ }^{44,45}$ To date, pre-transplant cardiac evaluation has been formally recommended by the European Society for Blood and Marrow (EBMT) and partners, and once incorporated by transplant centers, should contribute to a reduction in transplant-related mortality. ${ }^{20}$ In parallel, alternative conditioning regimens, including lower doses of cyclophosphamide or different non-cardiotoxic agents, are also under investigation. $^{24,46}$

Over the 20 past years, transplant-related mortality has declined from over $17 \%$ to less than $6 \%$, and some centers have reported zero deaths from the procedure. ${ }^{16,38,47}$ Systemic sclerosis is a complex disease and internal organ damage increases the toxicity of the procedure. 


\begin{tabular}{|c|c|c|c|c|c|c|c|}
\hline 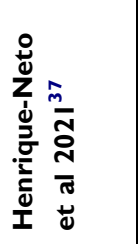 & $\begin{array}{l}\overline{\bar{N}} \\
\bar{y} \\
\bar{D}\end{array}$ & 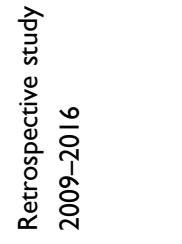 & 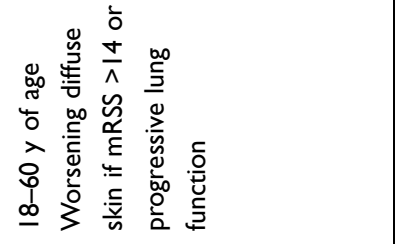 & 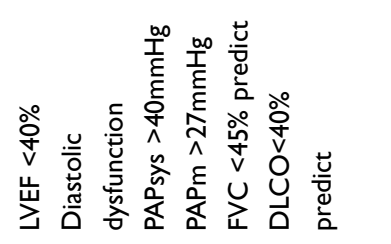 & $R$ & 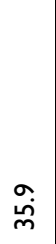 & $\vec{\lambda}$ \\
\hline 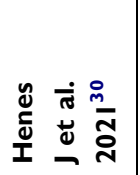 & 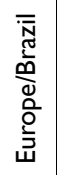 & 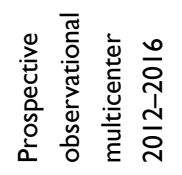 & 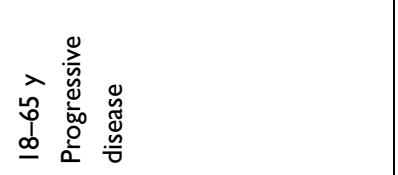 & & ๑ & 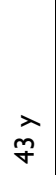 & 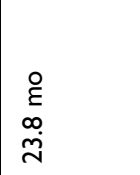 \\
\hline 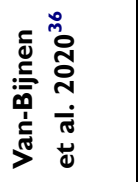 & 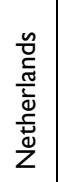 & 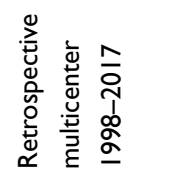 & 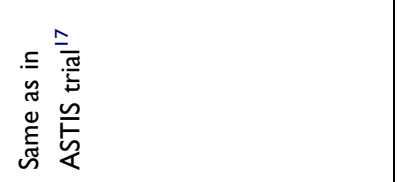 & 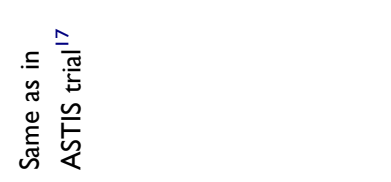 & ळ & $\hat{f}$ & $\begin{array}{c}\stackrel{\circ}{\xi} \\
\underline{\infty}\end{array}$ \\
\hline 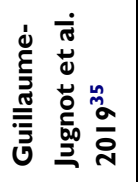 & 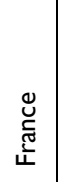 & 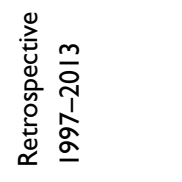 & ż & 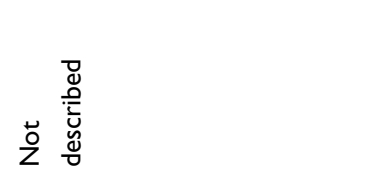 & மீ & $\underset{\stackrel{\infty}{+}}{\widehat{\infty}}$ & $\begin{array}{c}\stackrel{0}{\varepsilon} \\
\stackrel{n}{N}\end{array}$ \\
\hline 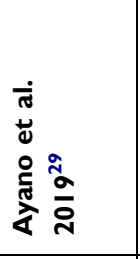 & 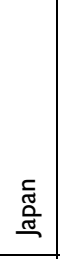 & 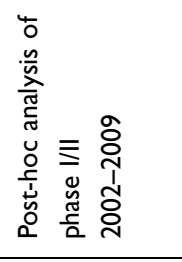 & 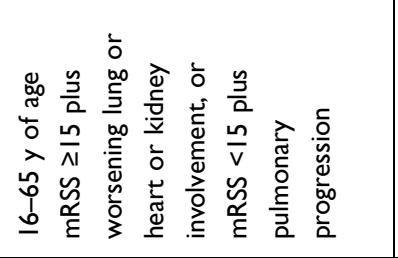 & 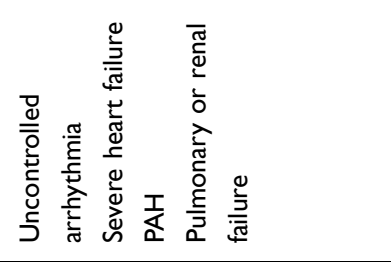 & $\underline{\alpha}$ & $\begin{array}{l}\hat{\lambda} \\
\hat{n}\end{array}$ & $\begin{array}{l}\stackrel{\circ}{g} \\
\text { 으 }\end{array}$ \\
\hline 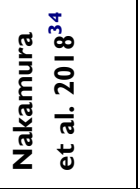 & 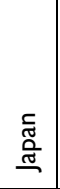 & 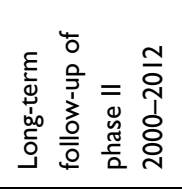 & 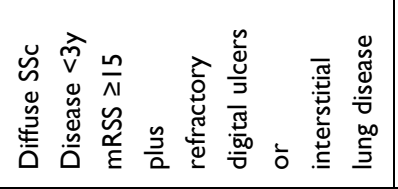 & 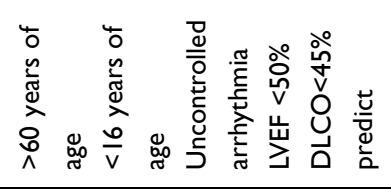 & \pm & $\stackrel{\substack{n \\
+}}{+}$ & 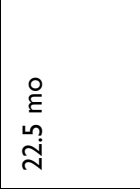 \\
\hline 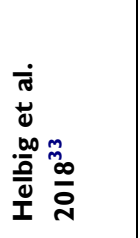 & $\begin{array}{l}\frac{7}{c} \\
\frac{\mathrm{c}}{0} \\
\mathrm{O}\end{array}$ & 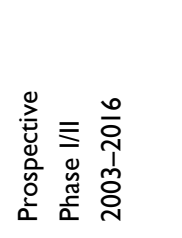 & 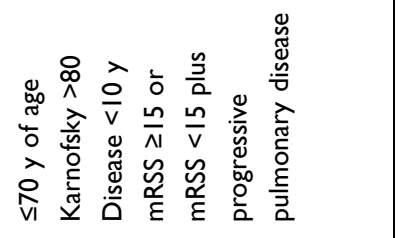 & 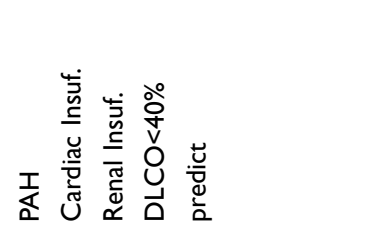 & $\underline{\infty}$ & $\frac{\lambda}{n}$ & 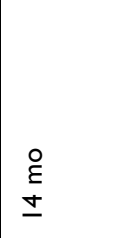 \\
\hline 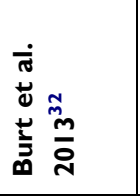 & $\begin{array}{l}\overline{\bar{d}} \\
\stackrel{\bar{m}}{m} \\
\substack{د \\
د} \\
\end{array}$ & 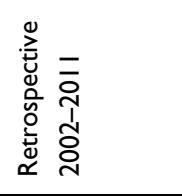 & 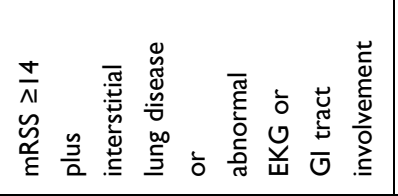 & 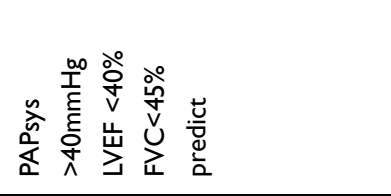 & \& & $\widehat{\mathcal{F}}$ & $\begin{array}{c}\stackrel{\circ}{\xi} \\
\stackrel{2}{\sim}\end{array}$ \\
\hline 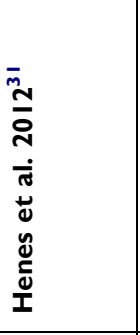 & 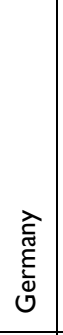 & 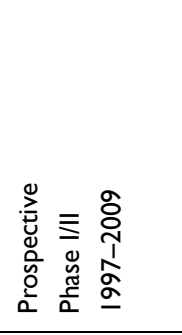 & 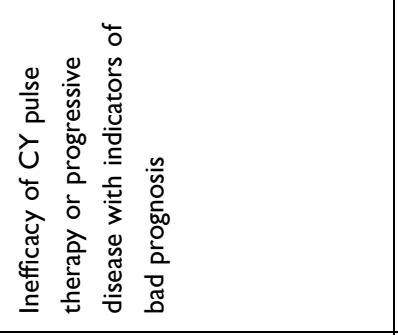 & 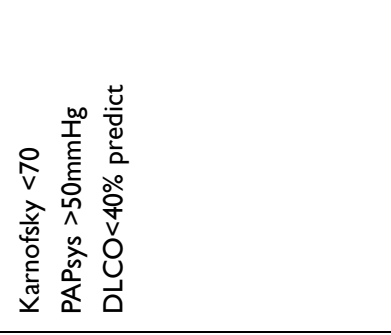 & $\stackrel{2}{\sim}$ & बे & $\hat{\lambda}$ \\
\hline & $\begin{array}{l}\text { ते } \\
\text { 产 } \\
\text { Uे }\end{array}$ & 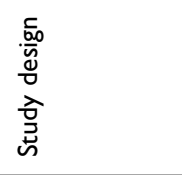 & 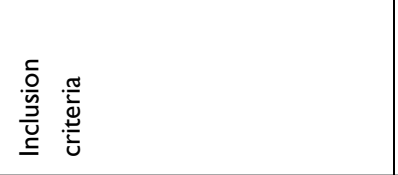 & 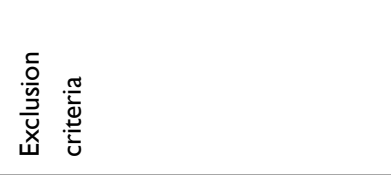 & $z$ & 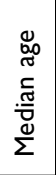 & 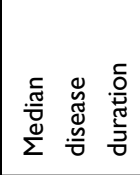 \\
\hline
\end{tabular}




\begin{tabular}{|c|c|c|c|c|c|c|c|c|c|}
\hline 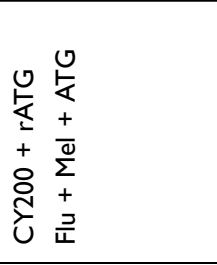 & z & $\widehat{\infty}$ & 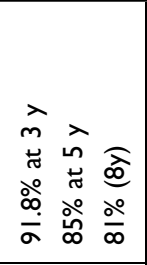 & $\begin{array}{l}\stackrel{\widehat{o}}{\frac{0}{m}} \\
\stackrel{\circ}{\dot{\sigma}}\end{array}$ & 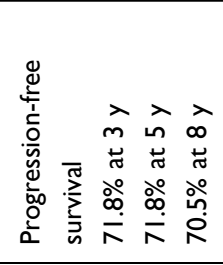 & ஓे & 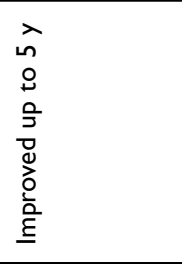 & 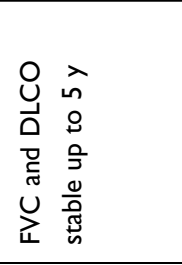 & 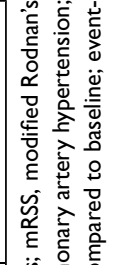 \\
\hline 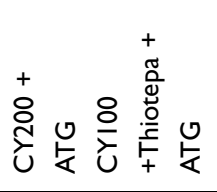 & 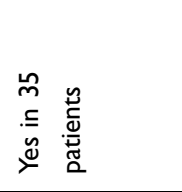 & $\vec{\lambda}$ & 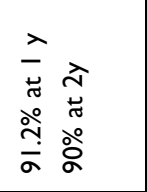 & $\begin{array}{l}\widehat{\circ} \\
\stackrel{\infty}{n} \\
\stackrel{\circ}{0}\end{array}$ & 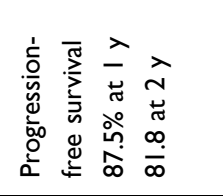 & 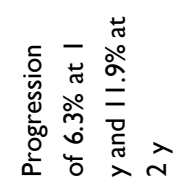 & 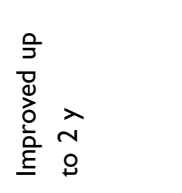 & 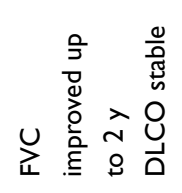 & 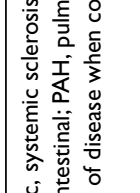 \\
\hline 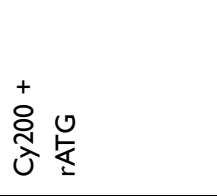 & $\stackrel{\check{\nu}}{\check{\nu}}$ & $\begin{array}{l}\hat{o} \\
\dot{+}\end{array}$ & 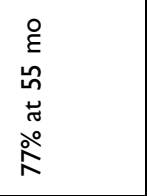 & $\begin{array}{l}\stackrel{\sigma}{\infty} \\
\stackrel{\infty}{\infty} \\
\stackrel{\circ}{=}\end{array}$ & 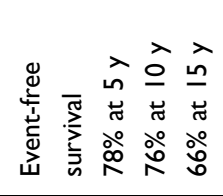 & 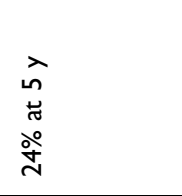 & 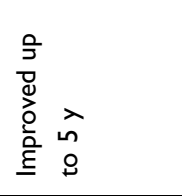 & 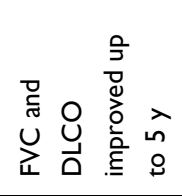 & 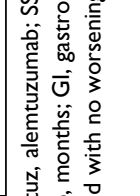 \\
\hline 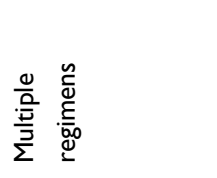 & 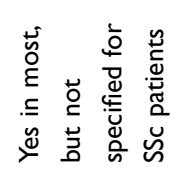 & $\vec{n}$ & 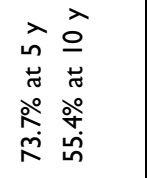 & 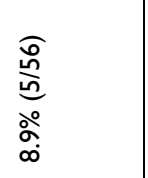 & 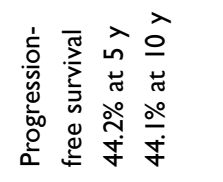 & 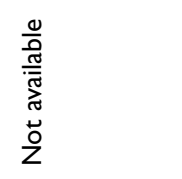 & 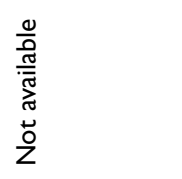 & 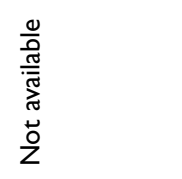 & 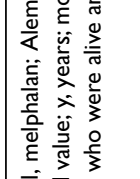 \\
\hline$\overbrace{i}^{8}$ & 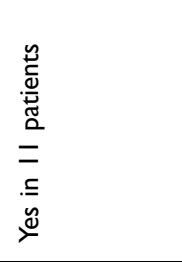 & $\widehat{\infty}$ & 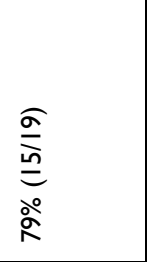 & $\stackrel{\circ}{\stackrel{\circ}{N}}$ & 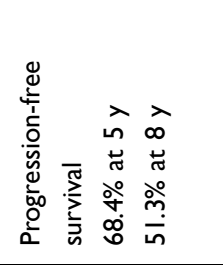 & $\begin{array}{l}\frac{0}{0} \\
\frac{0}{0} \\
\frac{\pi}{\pi} \\
\frac{\pi}{\pi} \\
\text { z } \\
z\end{array}$ & 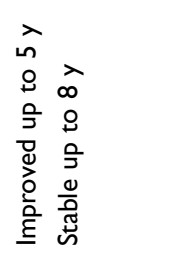 & 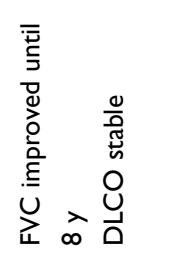 & 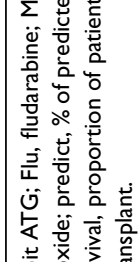 \\
\hline ¿্ণ & 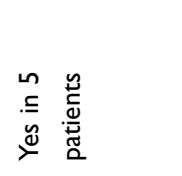 & $\underline{\underline{z}}$ & 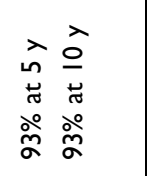 & 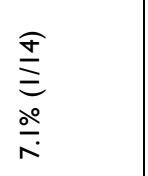 & 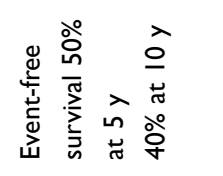 & 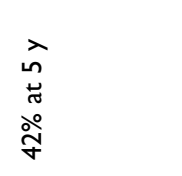 & 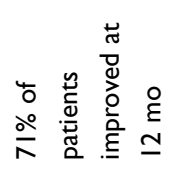 & 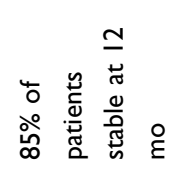 & 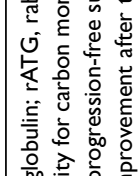 \\
\hline 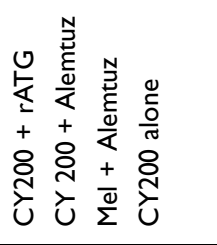 & z & $\begin{array}{c}\stackrel{o}{\xi} \\
\underset{f}{q}\end{array}$ & $\begin{array}{c}\varepsilon \\
\frac{g}{q} \\
\frac{U}{\sigma} \\
\frac{0}{6}\end{array}$ & 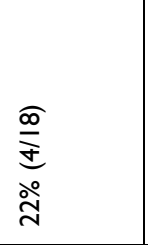 & 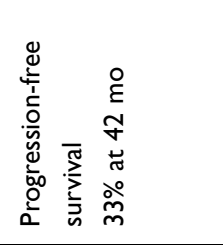 & 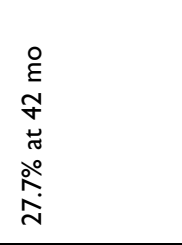 & 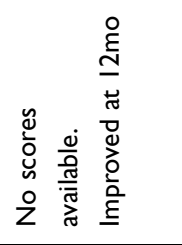 & 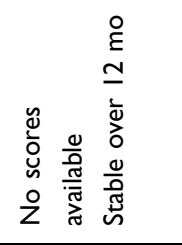 & 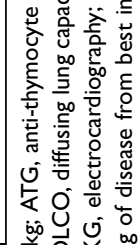 \\
\hline 荌 & \& & in & 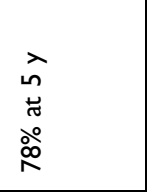 & 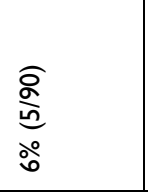 & 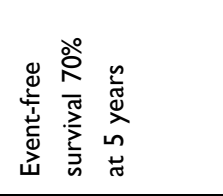 & 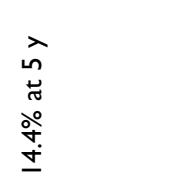 & 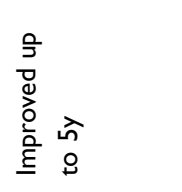 & 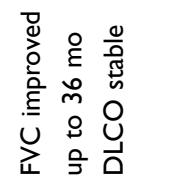 & 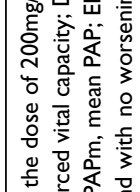 \\
\hline 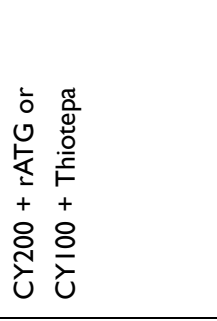 & $\stackrel{\tilde{y}}{\check{0}}$ & $\begin{array}{l}\hat{\vec{t}} \\
\overrightarrow{+}\end{array}$ & $\begin{array}{c}\lambda \\
m \\
\vdots \\
\vdots \\
\stackrel{े}{1}\end{array}$ & $\begin{array}{l}\stackrel{\widehat{g}}{=} \\
\stackrel{\circ}{+}\end{array}$ & 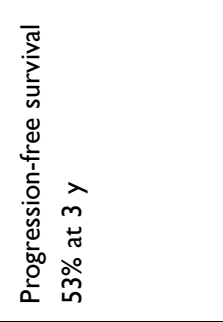 & $\begin{array}{l}\lambda \\
m \\
\text { जे } \\
\stackrel{0}{\circ} \\
\dot{j} \\
\dot{m}\end{array}$ & 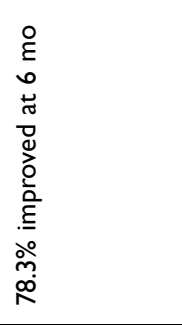 & 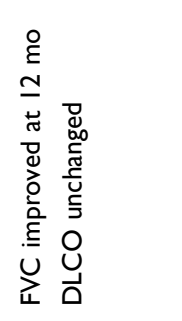 & 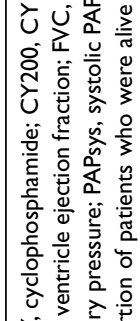 \\
\hline 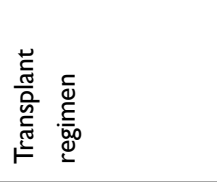 & 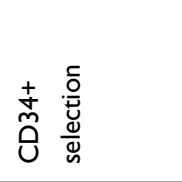 & 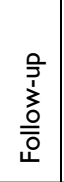 & 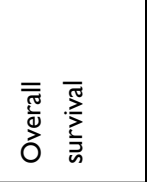 & 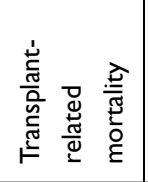 & 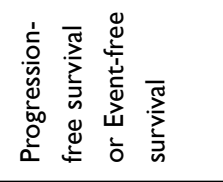 & 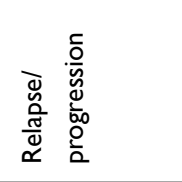 & $\begin{array}{l}\mathscr{W} \\
\stackrel{\widetilde{\alpha}}{\xi}\end{array}$ & 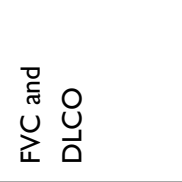 & 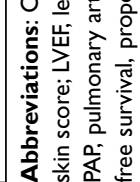 \\
\hline
\end{tabular}


Table 2 Main Characteristics of Comparative Studies, Including Randomized Controlled Trials

\begin{tabular}{|c|c|c|c|c|}
\hline & $\begin{array}{l}\text { Burt et al } 201 \mathrm{I} \\
\text { (ASSIST) }^{16}\end{array}$ & $\begin{array}{l}\text { van Laar et al } 2014 \\
\text { (ASTIS) }^{17}\end{array}$ & Sullivan et al 2018 (SCOT) $)^{18}$ & Del Papa et al. $2017^{38}$ \\
\hline Study design & $\begin{array}{c}\text { Phase II, randomized I:I, } \\
\text { open-label } \\
\text { cross-over to HSCT } \\
\text { allowed at } 12 \text { mo } \\
\text { North American single- } \\
\text { center } \\
\text { 2006-2009 }\end{array}$ & $\begin{array}{l}\text { Phase III, randomized I:I, } \\
\text { open-label } \\
\text { European multicenter } \\
\text { (EBMT) } \\
200 \mathrm{I}-2009\end{array}$ & $\begin{array}{l}\text { Phase II, randomized I:I, open- } \\
\text { label } \\
\text { North American multicenter } \\
\text { 2005-20II }\end{array}$ & $\begin{array}{l}\text { Phase II, retrospective } \\
\text { historical SSc control group } \\
\text { Italian, single-center } \\
2003-201 \text { I }\end{array}$ \\
\hline Comparisons & $\begin{array}{l}\mathrm{HSCT} \text { vs } 6-\mathrm{mo} \\
\text { intravenous } \mathrm{CY}\end{array}$ & $\begin{array}{l}\mathrm{HSCT} \text { vs } 12-\mathrm{mo} \\
\text { intravenous } \mathrm{CY}\end{array}$ & HSCT vs I2-mo intravenous CY & HSCT vs historical cohort \\
\hline $\begin{array}{l}\text { Inclusion } \\
\text { criteria }\end{array}$ & $\begin{array}{c}<60 \text { y of age } \\
\text { Disease duration } \leq 4 \mathrm{y} \\
\text { Diffuse SSc } \\
\text { mRSS } \geq 15 \\
\text { Internal organ } \\
\text { involvement }\end{array}$ & $\begin{array}{c}18-65 \text { y of age } \\
\text { Disease duration } \leq 2-4 y^{a} \\
\text { Diffuse SSc } \\
\text { mRSS } \geq 15 \\
\text { Internal organ involvement }\end{array}$ & $\begin{array}{c}\text { 18-69 y of age } \\
\text { Disease duration } \leq 4 \text { y } \\
\text { Diffuse SSc } \\
\text { mRSS } \geq 16 \\
\text { Internal organ involvement }\end{array}$ & $\begin{array}{c}\text { Diffuse SSc } \\
\text { Disease duration } \leq 4 \text { y } \\
m R S S \geq 14 \\
\text { Clinical activity score (ESSG) } \geq 3\end{array}$ \\
\hline $\begin{array}{l}\text { Exclusion } \\
\text { criteria }\end{array}$ & $\begin{array}{c}\text { PAPm }>25 \mathrm{mmHg} \text { or } \\
\text { PAPsys }>40 \mathrm{mmHg} \\
\text { LVEF }<40 \% \\
\text { Creatinine }>177 \mu \mathrm{mol} / \mathrm{L} \\
>6 \text { Intravenous CY } \\
\text { courses }\end{array}$ & $\begin{array}{c}\text { PAPm }>50 \mathrm{mmHg} \\
\text { LVEF }<45 \% \\
\text { Creatinine }>40 \mathrm{~mL} / \mathrm{min} \\
\text { Cumulative IV } \mathrm{CY} \text { dose } \\
>5 \mathrm{~g} \text { or Cumulative oral } \mathrm{CY} \\
\text { dose }>3 \mathrm{~g}\end{array}$ & $\begin{array}{c}\text { Mean PAP }>30 \mathrm{mmHg} \\
\text { LVEF }<50 \% \\
\mathrm{FVC}<45 \% \text { predicted } \\
\text { DLCO }<40 \% \text { predicted } \\
\text { Creatinine clearance }<40 \mathrm{~mL} / \mathrm{min} \\
\text { Cumulative IV CY dose }>3 \mathrm{~g} / \mathrm{m}^{2} \\
\text { or } \\
\text { Oral CY dose }>4 \text { months, or } \\
>6 \text { Intravenous } \mathrm{CY} \text { courses }\end{array}$ & $\begin{array}{c}\text { PAH } \\
\text { LVEF }<45 \% \\
\text { DLCO }<50 \% \text { predicted, } \\
\text { Prior renal crisis }\end{array}$ \\
\hline $\begin{array}{l}\text { Number of } \\
\text { participants }\end{array}$ & $\begin{array}{c}19(10 \mathrm{HSCT}+9 \mathrm{CY} \\
\text { arm })\end{array}$ & $\begin{array}{c}\text { I56 (79 HSCT }+77 \mathrm{CY} \\
\text { arm })\end{array}$ & 75 (33 HSCT + 32 CY arm) & $18 \mathrm{HSCT}+36 \mathrm{SSc}$ controls \\
\hline Median age & $45 y$ & $43.8 y$ & $45.9 y$ & 41 y \\
\hline $\begin{array}{l}\text { Disease } \\
\text { duration }\end{array}$ & $13.6 \mathrm{mo}$ & $16.8 \mathrm{mo}$ & $27 \mathrm{mo}$ & $24 \mathrm{mo}$ \\
\hline $\begin{array}{l}\text { Transplant } \\
\text { regimen }\end{array}$ & CY200 + rabbit ATG & CY200 + rabbit ATG & TBI + CYI20 + equine ATG & CY200 + rabbit ATG \\
\hline $\begin{array}{l}\text { Total body } \\
\text { irradiation }\end{array}$ & No & No & Yes & No \\
\hline $\begin{array}{l}\text { CD34+ } \\
\text { selection }\end{array}$ & No & Yes & Yes & Yes \\
\hline $\begin{array}{l}\text { Follow-up } \\
\text { after AHSCT }\end{array}$ & 2.6 y (median) & 5.8 y (median) & 4.5 y (minimum) & 5 y (minimum) \\
\hline $\begin{array}{l}\text { Overall } \\
\text { survival }\end{array}$ & $\begin{array}{c}100 \% \text { at median } 2.6 \text { y for } \\
\text { both groups }\end{array}$ & $\begin{array}{l}80 \% \text { in HSCT vs } 65 \% \text { in CY } \\
\text { at } 4 \text { y } \\
75 \% \text { in HSCT vs } 60 \% \text { in CY } \\
\text { at } 8 y\end{array}$ & $86 \%$ in HSCT vs $51 \%$ in CYat $6 y$ & $\begin{array}{c}89 \% \text { in HSCT vs } 39 \% \text { in SSc } \\
\text { controls at } 5 y\end{array}$ \\
\hline $\begin{array}{l}\text { Transplant- } \\
\text { related } \\
\text { mortality }\end{array}$ & $0 \%$ & $10.6 \%(8 / 79$ HSCT $)$ & $3 \%(\mathrm{I} / 33 \mathrm{HSCT})$ & $5.6 \%(1 / 18 \mathrm{HSCT})$ \\
\hline
\end{tabular}

(Continued) 
Table 2 (Continued).

\begin{tabular}{|c|c|c|c|c|}
\hline & $\begin{array}{l}\text { Burt et al } 20 I I \\
(\text { ASSIST) }\end{array}$ & $\begin{array}{l}\text { van Laar et al } 2014 \\
\text { (ASTIS) }^{17}\end{array}$ & Sullivan et al 2018 (SCOT) $^{18}$ & Del Papa et al. $2017^{38}$ \\
\hline $\begin{array}{l}\text { Event-free } \\
\text { survival }\end{array}$ & $\begin{array}{l}80 \% \text { in the HSCT group } \\
\text { at } 2 y \\
11 \% \text { in the CY group at } 2 \\
y\end{array}$ & $\begin{array}{c}81 \% \text { in } \mathrm{HSCT} \text { vs } 74 \% \text { in } \mathrm{CY} \\
\text { at } 4 \mathrm{y}\end{array}$ & $\begin{array}{c}79 \% \text { in HSCT vs } 50 \% \text { in CY at } 4.5 \\
y \\
74 \% \text { in HSCT vs } 47 \% \text { in CY at } 6 \text { y }\end{array}$ & Not described \\
\hline $\begin{array}{l}\text { Progression- } \\
\text { free survival }\end{array}$ & $\begin{array}{l}100 \% \text { in } \mathrm{HSCT} \text { vs } 11 \% \\
\mathrm{CY} \text { at I y } \\
88 \% \text { in } \mathrm{HSCT} \text { at } 2.6 \mathrm{y}\end{array}$ & $\begin{array}{c}77 \% \text { in HSCT vs } 65 \% \text { in CY } \\
\text { at } 5.8 \mathrm{y}\end{array}$ & Not described & $\begin{array}{l}\text { Not available. Higher survival in } \\
\text { HSCT than in the SSc control } \\
\text { group }\end{array}$ \\
\hline $\begin{array}{l}\text { Disease } \\
\text { progression }\end{array}$ & $\begin{array}{c}0 \text { in } \mathrm{HSCT} \text { vs } 89 \%(8 / 9) \\
\text { in CY at I y }\end{array}$ & $\begin{array}{c}1 \mathrm{I} \% \text { in HSCT vs } 35 \% \text { in } \\
\text { CYC at } 5.8 \mathrm{y}\end{array}$ & $\begin{array}{c}18 \% \text { in } \mathrm{HSCT} \text { vs } 41 \% \text { in } \mathrm{CY} \text { at } 6 \\
\text { years }\end{array}$ & $\begin{array}{l}\text { Not available. Lower disease } \\
\text { progression in HSCT than in } \\
\text { controls }\end{array}$ \\
\hline mRSS & $\begin{array}{l}\text { Improvement up to } 2 \mathrm{y} \text { in } \\
\qquad \mathrm{HSCT} \\
\text { Worsening in } \mathrm{CY} \text { group }\end{array}$ & $\begin{array}{c}\text { Improvement at } 2 y \text { in } \\
\text { HSCT } \\
\text { HSCT better than CY at } 2 \\
y\end{array}$ & Not described & $\begin{array}{c}\text { Improvement from baseline to } 12 \\
\text { mo after HSCT and stabilization } \\
\text { thereafter }\end{array}$ \\
\hline FVC/DLCO & $\begin{array}{l}\text { FVC improved more in } \\
\text { HSCT than in CY } \\
\text { DLCO remained stable } \\
\text { and not different } \\
\text { between groups }\end{array}$ & $\begin{array}{c}\text { FVC improved more in } \\
\text { HSCT than CY } \\
\text { DLCO remained stable and } \\
\text { not different between } \\
\text { groups }\end{array}$ & $\begin{array}{c}\text { FVC improved/stabilized in more } \\
\text { patients from HSCT than from } \\
\text { CY group }\end{array}$ & $\begin{array}{l}\text { Stabilization of FVC and DLCO } \\
\text { No difference between HSCT and } \\
\text { controls }\end{array}$ \\
\hline
\end{tabular}

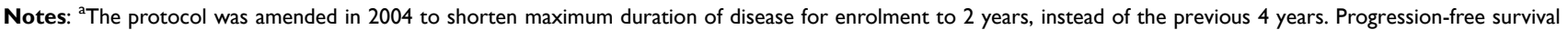
(PFS): proportion of patients who were alive and with no worsening of disease when compared to baseline; event-free survival: proportion of patients who were alive and with no worsening of disease from best improvement after transplant.

Abbreviations: SSc, systemic sclerosis; HSCT, hematopoietic stem cell transplantation; mRSS, modified Rodnan's skin score; ESSG, European Scleroderma Study Group scoring system; LVEF, left ventricle ejection fraction; PAP, pulmonary artery pressure; PAPsys, systolic PAP; PAPm, mean PAP; CY, cyclophosphamide; CY200, CY at a dose of $200 \mathrm{mg} / \mathrm{kg}$; ATG, anti-thymocyte globulin; TBI, total body irradiation; PAH, pulmonary artery hypertension; FVC, forced vital capacity; DLCO, diffusing lung capacity for carbon monoxide; $y$, years; mo, months.

Therefore, the expertise of the transplant team in selecting the appropriate patients, and thereby excluding those with too advanced organ damage, and in managing intratransplant events and complications, is essential for good patient outcomes. An EBMT study from 2010 retrospectively analyzed data from multiple autoimmune disease transplants, showing that less experienced transplant centers, ie, those with lower number of transplanted patients, had higher transplant-related deaths. These results indicate that there is an important learning curve associated with outcomes, especially considering that $\mathrm{SSc}$ is a disease difficult to manage during AHSCT. ${ }^{48}$

Tissue damage is accumulated over time in SSc. ${ }^{49}$ Patients earlier in disease course have less internal organ impairment and should thus present less transplant-related toxicity and perhaps better disease control after AHSCT. In line with this, recent prospective studies have included patients with disease duration limited to 2 to 4 years. ${ }^{16-18,38}$ Indeed, a very recent prospective study conducted by the EBMT showed, by multivariate analysis, that mRSS above 24 at baseline and older age at transplantation were significantly associated with lower progression-free survival, corroborating that patients should be enrolled earlier in disease course. ${ }^{30}$ The contribution of this selection strategy to the final patient outcome is unknown, but an ongoing study that aims to enroll patients for AHSCT as upfront treatment and, therefore, shortly after diagnosis, should answer some of the pending questions. $^{50}$

\section{Disease Progression}

Studies are heterogeneous on reports of disease control after AHSCT. Nevertheless, most studies demonstrate positive effects of AHSCT on patient outcomes. Disease progression, defined as worsening manifestations from baseline, varied between the phase I/II non-randomized studies, even between those with similar duration of follow-up. Disease progression rates varied from $11.9 \%$ at 2 years to $30.4 \%$ at 4.4 years after ASHCT. ${ }^{30,31}$ For studies 
with longer follow-up, the 8-year progression rate ranged from $10.5 \%$ to $24 \%,{ }^{29,37}$ reaching $42 \%$ at 11 years. $^{34}$ These different rates are probably secondary to differences in patient selection, different criteria for progression and duration of follow-up.

The three randomized studies - ASSIST, ASTIS and SCOT - showed better disease control in transplanted versus conventionally treated (control) patients. ${ }^{16-18}$ This difference in event-free survival between transplanted and control groups is more pronounced in the SCOT $(74 \%$ in transplanted vs $47 \%$ in controls), than in the ASTIS trial (77\% in transplanted vs $65 \%$ in controls), probably owing to the higher transplant-related mortality of ASTIS and to slightly different criteria for disease progression between studies. In the SCOT trial, the progression rate was not described in traditional terms, as patient outcomes were measured by a specific global rank composite score (GRCS). This score included a hierarchy of events reflecting disease improvement or worsening, resulting in a final score that enabled comparisons between participants of the study. Unfortunately, the evaluating method does not allow outcomes to be directly compared to those from other studies. Nevertheless, a recent study retrospectively evaluated the French cohort of the ASTIS trial, consisting of 49 patients, for GRCS results, showing superiority of transplanted versus conventionally treated patients. ${ }^{51}$ In this study, GRCS was 9 for transplanted versus -19 for conventionally treated patients at 60 months $(p=0.018)$, while in SCOT, respective results were 20 and -8 at 48 months $(p=0.008)$.

\section{Skin Outcomes}

Clinical trials on AHSCT for SSc show a significant reduction in skin thickening, assessed by the modified Rodnan skin score (mRSS). ${ }^{52}$ Improvement in skin involvement is considered when mRSS decreases by more than $25 \%{ }^{16,18,30}$ Collectively, phase I/II studies show improvement of mRSS early after AHSCT, usually more pronounced within the first year after transplantation, and tending to stabilize thereafter. ${ }^{16,30,32,37}$ Longer follow-up studies show sustained mRSS improvement for at least 60 and 96 months, respectively. ${ }^{29,37}$ In a post-hoc analysis of a phase I/II non-randomized study, patients who underwent AHSCT with and without graft selection were compared. CD34+ graft selection had an overall positive influence on patient outcomes but was specifically associated with improvements in $\mathrm{mRSS}^{29}$
In the randomized controlled studies, the differences in mRSS between patients undergoing AHSCT and those treated with cyclophosphamide (control) are significant, usually with improving curves in transplanted and progressively worsening in control patients. In ASSIST, mRSS improved from 30 at baseline, to 16 at 12 months, and 9 at 24 months after AHSCT, while scores worsened in the control group. Patients from the control group were allowed to cross over to the transplantation group after 1 year of follow-up, and these also had improvements in mRSS. ${ }^{16}$ The ASTIS and SCOT trials also show superior efficacy of transplanted over control patients regarding skin involvement, with sustained outcomes at 24 and 54 months, respectivelly. ${ }^{17,18}$

In a non-randomized, but comparative study, both mRSS and European Scleroderma Study Group (ESSG) scores showed a strongly significant reduction since 12 months after AHSCT. When groups were compared at 3-years, the probability that mRSS could decline to below 14 was above $90 \%$ in the AHSCT group, while only $60 \%$ in the control group. ${ }^{38}$

Improvement of the skin involvement may be also indirectly assessed by functional evaluations, such as joint range-of-motion measurements, hand grip strenght, finger-to-palm distance, mouth opening, and functional questionnaires for hand (Cochin) and upper limbs (DASH, Disabilities of the arm, shoulder and hand). A recent study showed improvements of these functional parameters at 6 and 12 months after AHSCT, when compared to baseline. ${ }^{53}$

Although the mRSS is the universally used method to quantify skin thickening in SSc, it bears intrinsic limitations and depends on evaluator expertise and opinion that affect reproducibility and consistency of the method. ${ }^{54}$ Therefore, the reliability of mRSS to evaluate skin outcomes after AHSCT has been questioned. Nevertheless, skin biopsies, the gold-standard method to quantify cutaneous involvement in SSc, correlate well with mRSS, as the degree of fibrosis decreases while mRSS scores decline after AHSCT. ${ }^{14,55}$

\section{Pulmonary Outcomes}

Most phase I/II studies showed stabilization or slight improvement in FVC after transplantation, and DLCO stabilization. $^{30-33}$ One study showed significant improvement for both FVC and DLCO over 5 years and another study showed that FVC and DLCO improved only in the subgroup of patients with progressive lung disease as an 
indication for AHSCT. ${ }^{36,37}$ The ASSIST and ASTIS randomized trials showed that lung function outcomes in transplanted patients were superior to those from the control group treated with cyclophosphamide. ${ }^{16,17}$ In ASSIST, in addition to the favorable difference between groups, $\mathrm{CVF}$ and computed tomography volumes increased in transplanted patients at 24 months of follow-up. ${ }^{16}$ The SCOT study does not describe pulmonary function data in a format that enables comparisons, but there is a higher absolute number of patients having improved FVC after AHSCT than after cyclophosphamide pulses. ${ }^{18}$

A retrospective study from the EBMT did not associate graft selection with clinical outcomes, including pulmonary function test results. ${ }^{28} \mathrm{~A}$ more recent study, however, prospectively compared two groups of SSc patients, randomized for AHSCT with manipulated or non-manipulated graft. ${ }^{29}$ The authors showed that although overall survival was similar between the two groups, patients who received $\mathrm{CD} 34+$ selected grafts had better progression-free survival over an 8-year follow-up. Forced vital capacity also progressively increased over the years in the CD34+ selected patients, while remained mostly stable in the non-selected group, with a tendency to better outcomes in the selected versus non-selected group.

Physical capacity, assessed by the 6-minute walk test (6MWT), improved after AHSCT, as an indirect evidence of better pulmonary function, although the cardiovascular and musculoskeletal systems may have participated. ${ }^{53}$ Another method to evaluate how AHSCT affects interstitial lung disease is high-resolution quantitative computed tomography. Studies have shown improvement of lung volumes and/or pulmonary tissue quality (evaluated by density), after transplantation, associated with pulmonary function outcomes. ${ }^{16,56-58}$

\section{Quality of Life}

Quality of life is an important indicator of transplant outcomes, as it reflects the patient's perspective and evaluates how patients are affected by the procedure in the context of their daily lives and environment. Quality of life assessments have been included in several clinical trials, but only one study has specifically addressed this aspect of evaluation in SSc patients undergoing AHSCT. ${ }^{59}$ This retrospective study compared Short-Form 36 (SF36) questionnaire results from $41 \mathrm{SSc}$ patients who underwent AHSCT and 65 conventionally treated (control) SSc patients, over a 7-year follow-up. Patient groups were different for physical components of quality of life, favoring transplant, but no difference was detected between groups regarding the mental components of quality of life. In addition, Health Assessment Questionnaire (HAQ) scores were considerably lower (indicating better function) at all times in patients treated with AHSCT compared to the control group. ${ }^{59}$

Recently, a prospective, open and multicenter study assessed Scleroderma HAQ (sHAQ) scores in 15 patients who underwent AHSCT, showing significant improvement at 1 year and and 2 years of follow-up. ${ }^{30}$ Another singlecenter study also prospectively evaluated quality of life in 28 SSc patients treated with AHSCT, showing improvement of the physical components of SF36 at 6 and 12 months, and of the mental components of SF36 at 12 months. ${ }^{53}$ Finally, a systematic review from 2020 analyzed three randomized and five uncontrolled clinical trials that contained information about quality of life in transplanted SSc patients, showing that SF36 results were heterogeneous across studies, but with overall improvements in the physical components of quality of life. For the mental components, however, data were inconsistent. ${ }^{60}$

\section{Fertility After AHSCT}

Fertility is usually preserved in SSc patients, as there are no differences in rates of conception between SSc women and the general healthy population. ${ }^{61,62}$ In the subset of cyclophosphamide-treated patients, however, fertility rates may be compromised, due to gonadal toxicity of the treatment, especially in women. ${ }^{63}$ Pregnancies, on the other hand, may have worse outcomes in SSc women than in the non-SSc patients. ${ }^{61}$ An Italian study has retrospectively evaluated 109 pregnancies in SSc women, showing a higher rate of preterm deliveries, intrauterine growth restriction and very low-weight babies than in the general obstetric population. ${ }^{62}$ Male fertility is much less investigated and few studies have specifically addressed male impotency in SSc. ${ }^{64,65}$

In the setting of AHSCT, reduced fertility is a frequently reported complication, both in men and women, although SSc data are reported combined with other autoimmune diseases. Multicenter data from the EBMT that were retrospectively analyzed showed that among 324 adult female patients with autoimmune diseases that underwent AHSCT, 15 of them had 22 pregnancies along post-transplantation follow-up. Five of these patients had SSc as baseline disease, and all had received high-dose cyclophosphamide as part of the transplantconditioning regimen. One of the SSc patients had three 
pregnancies, including one miscarriage. There were no deaths during pregnancy, but one patient died shortly after delivery due to worsening of skin and pulmonary fibrosis. ${ }^{66}$

A single center from Germany described 15 patients (11 female), who had been previously treated with autologous AHSCT for severe autoimmune diseases, out of which 3 had SSc. All but one patient had received cyclophosphamide as conventional treatment prior to AHSCT and all received high-dose cyclophosphamide as part of transplant conditioning regimen. One female patient with SSc was considered to have impaired fertility since before AHSCT, remaining amenorrheic and not becoming pregnant after AHSCT. Another female SSc patient became temporarily amenorrheic shortly after AHSCT, recovering regular menses a few months later. This woman became spontaneously pregnant twice after AHSCT, with successful preterm deliveries at 34 and 35 weeks due to premature labour and breech presentation, respectively. ${ }^{67}$

\section{Secondary Autoimmune Diseases After AHSCT}

A debated concern in the field of AHSCT for autoimmune diseases is whether the profound manipulation of the immune system in genetically predisposed patients may lead to development of secondary autoimmune diseases. ${ }^{68}$ Therefore, continuous long-term surveillance of posttransplant immune status is recommended for patients who undergo AHSCT.

A study from the EBMT has reported a cumulative incidence of secondary autoimmune diseases of 9.8\% over a 5-year follow-up after AHSCT, among 347 patients who underwent AHSCT with an autoimmune disease as primary indication. ${ }^{69}$ Most secondary autoimmune diseases were organ or tissue-specific, with variable severity. Two, out of the 29 patients who developed secondary autoimmune diseases after AHSCT, died as direct consequence of antiphospholipid syndrome (cerebral ischemia) and hemorrhage (acquired hemophylia), respectively. Multivariate analysis identified systemic lupus erythematosus as primary indication for AHSCT and use of antithymocyte globulin associated with graft selection in the conditioning regimen as risk factors for development of secondary autoimmune disease. ${ }^{69}$

Additional secondary autoimmune diseases have been reported in smaller case series. One, out of 26 transplanted $\mathrm{SSc}$ patients, presented morphea as a secondary autoimmune manifestation after AHSCT. ${ }^{31}$ In a cohort of 14 Japanese SSc patients treated with AHSCT, one patient developed multiple overlapped autoimmune disorders including Grave's disease, immune thrombocytopenia, systemic lupus erythematosus and antiphospholipid syndrome, and a second patient developed Sjogren's syndrome. ${ }^{34}$ In a case series of $6 \mathrm{SSc}$ patients who underwent AHSCT with thiotepa as part of the conditioning regimen, two female patients developed symptomatic Grave's disease with detectable autoantibodies against thyroid stimulating hormone (TSH) receptor, at 13 and 19 months after AHSCT. A third male patient developed antibodies against SSA and polyclonal hypergammaglobulinaemia 6 months after transplantation. ${ }^{24}$ Finally, a French long-term outcome study reported secondary autoimmune diseases in $5(8.9 \%)$ out of 56 transplanted SSc patients; thyroiditis, autoimmune hemolytic anemia, myasthenia gravis, sarcoidosis and anti-phospholipid syndrome. ${ }^{35}$

\section{Secondary Malignancies After AHSCT}

Systemic sclerosis is associated with increased relative risk of cancer, mainly lung, liver, hematologic and bladder, although with a low absolute risk. ${ }^{70}$ Multiple mechanisms may contribute to such outcomes, including genetic background, defective immunological surveillance, pro-inflammatory status, epithelial hyperplasia and prior exposure to carcinogens, including those associated with cytotoxic treatment. ${ }^{71}$

Data on secondary malignancies in SSc after AHSCT are limited and variable. In addition, it is difficult to establish how much is influenced by the transplant procedure itself or by previous immunosuppressive and cytotoxic treatment, as well as prior viral infections. The ASTIS trial reported only one patient developed EpsteinBarr virus-related post-transplant lymphoproliferative disease shortly after AHSCT. Other five non-transplanted SSc patients from the cyclophosphamide (control) group also developed malignancies. ${ }^{17}$ In the SCOT study, 3 of $33 \mathrm{SSc}$ patients from the transplant group, and one from the conventional-treatment control group developed cancer. The authors believe that the regimen including total body irradiation may have contributed to the increased incidence of malignancy in the transplanted patients. ${ }^{18}$ In a retrospective evaluation, the French Society for Bone Marrow Transplantation and Cellular Therapy reported 4 (7\%) of $56 \mathrm{SSc}$ patients who underwent AHSCT and developed cancer (oesophagus epidermoid carcinoma, 
unspecified carcinoma, lung epidermoid carcinoma, spinocellular carcinoma) over a median follow-up of 83 months. ${ }^{35}$ Collectively, these results indicate that these patients may require longer follow-up and more detailed inspection for possible malignancies. Further studies should define if other factors, such as smoking or previous treatments, impose additional risks.

\section{Future Developments}

Although the field of AHSCT for SSc has grown over the years, translating into benefits for the patient, further improvements are still warranted. Strategies to discriminate subjects that may achieve best results after AHSCT may contribute to better outcomes and to consolidate the procedure as a therapeutic alternative for SSc. In this context, a study analyzing biological samples from participants of the SCOT trial clustered patients into groups, according to different gene expression profiles associated with different pathogenic mechanisms of disease. When each of these groups were analysed for clinical outcomes after AHSCT, patients with a more fibroproliferative profile showed the most significant long-term benefit, indicating a potential biomarker for patient selection before AHSCT. $^{72}$ More recently, this same research group was able to associate histological findings of fibroblast polarization with the previously defined fibroproliferative gene profile, and to further correlate them with severity of skin involvement assessed by mRSS. ${ }^{73}$ In addition, ongoing prospective clinical trials aim to evaluate the role of post-transplant maintenance therapy with immunosuppressors in decreasing disease reactivation and progression after AHSCT. ${ }^{74,75}$ Furthermore, more specific approaches to lessen transplant-related toxicity have been investigated. A recent strategy, still mostly limited to oncology and hematology fields, describes the use of antibody-targeted destruction of specific cell types. $^{76,77}$ Conjugation of toxins to anti-CD45 antibody, for instance, may concentrate depletion in hematopoietic cells and, thus, spare non-hematopoietic cells. This may be a future strategy for AHSCT in SSc patients, aiming to reduce transplant-related toxicity. Combined, clinical and translational activities are essential to develop the field, and to have patient welfare as the most important goal.

\section{Disclosure}

The authors report no conflicts of interest for this work.

\section{References}

1. Matucci-Cerinic M, Miniati I, Denton CP. Eular compendium on rheumatic diseases. In: Bijlsman JWJ, editor. Systemic Sclerosis. 2009:290-296.

2. Elhai M, Meune C, Boubaya M, et al. Mapping and predicting mortality from systemic sclerosis. Ann Rheum Dis. 2017;76 (11):1897-1905. doi:10.1136/annrheumdis-2017-211448

3. McMahan ZH, Volkmann ER. An update on the pharmacotherapeutic options and treatment strategies for systemic sclerosis. Expert Opin Pharmacother. 2020;21(16):2041-2056. doi:10.1080/ 14656566.2020.1793960

4. Elhai M, Meune C, Avouac J, Kahan A, Allanore Y. Trends in mortality in patients with systemic sclerosis over 40 years: a systematic review and meta-analysis of cohort studies. Rheumatology. 2012;51(6):1017-1026. doi:10.1093/rheumatology/ ker269

5. Topal AA, Dhurat RS. Scleroderma therapy: clinical overview of current trends and future perspective. Rheumatol Int. 2013;33 (1):1-18.

6. Tyndall AJ, Bannert B, Vonk M, et al. Causes and risk factors for death in systemic sclerosis: a study from the EULAR scleroderma trials and research (EUSTAR) database. Ann Rheum Dis. 2010;69 (10):1809-1815. doi:10.1136/ard.2009.114264

7. Fransen J, Popa-Diaconi D, Hesselstrand R, et al. Clinical prediction of 5-year survival in systemic sclerosis: validation of a simple prognostic model in EUSTAR centres. Ann Rheum Dis. 2011;70 (10):1788-1792. doi:10.1136/ard.2010.144360

8. Domsic RT, Medsger TA Jr. Connective tissue disease: predicting death in SSc: planning and cooperation are needed. Nat Rev Rheumatol. 2011;7(11):628-630. doi:10.1038/nrrheum.2011.152

9. Tyndall A. Hematopoietic stem cell transplantation for systemic sclerosis: review of current status. BioDrugs. 2019;33(4):401-409. doi:10.1007/s40259-019-00364-3

10. Marmont AM. Stem cell transplantation for autoimmune disorders. Coincidental autoimmune disease in patients transplanted for conventional indications. Best Pract Res Clin Haematol. 2004;17 (2):223-232. doi:10.1016/j.beha.2004.04.004

11. Binks M, Passweg JR, Furst D, et al. Phase I/II trial of autologous stem cell transplantation in systemic sclerosis: procedure related mortality and impact on skin disease. Ann Rheum Dis. 2001;60 (6):577-584. doi:10.1136/ard.60.6.577

12. Farge D, Marolleau JP, Zohar S, et al. Autologous bone marrow transplantation in the treatment of refractory systemic sclerosis: early results from a French multicentre Phase I-II study. $\mathrm{Br}$ $J \quad$ Haematol. 2002;119(3):726-739. doi:10.1046/j.13652141.2002.03895.x

13. Farge D, Passweg J, van Laar JM, et al. Autologous stem cell transplantation in the treatment of systemic sclerosis: report from the EBMT/EULAR Registry. Ann Rheum Dis. 2004;63(8):974-981. doi:10.1136/ard.2003.011205

14. Nash RA, McSweeney PA, Crofford LJ, et al. High-dose immunosuppressive therapy and autologous hematopoietic cell transplantation for severe systemic sclerosis: long-term follow-up of the US multicenter pilot study. Blood. 2007;110(4):1388-1396. doi:10.1182/ blood-2007-02-072389

15. Oyama Y, Barr WG, Statkute L, et al. Autologous non-myeloablative hematopoietic stem cell transplantation in patients with systemic sclerosis. Bone Marrow Transplant. 2007;40(6):549-555. doi:10.1038/sj.bmt.1705782

16. Burt RK, Shah S, Dill K, et al. Autologous non-myeloablative haemopoietic stem-cell transplantation compared with pulse cyclophosphamide once per month for systemic sclerosis (ASSIST): an open-label, randomised Phase 2 trial. Lancet. 2011;378 (9790):498-506. doi:10.1016/S0140-6736(11)60982-3 
17. Van Laar JM, Farge D, Sont JK, et al. Autologous hematopoietic stem cell transplantation vs intravenous pulse cyclophosphamide in diffuse cutaneous systemic sclerosis: a randomized clinical trial. JAMA. 2014;311(24):2490-2498. doi:10.1001/jama.2014.6368

18. Sullivan KM, Goldmuntz EA, Keyes-Elstein L, et al. Myeloablative autologous stem-cell transplantation for severe scleroderma. $N$ Engl $J$ Med. 2018;378(1):35-47. doi:10.1056/NEJMoa1703327

19. Kowal-Bielecka O, Fransen J, Avouac J, et al. Update of EULAR recommendations for the treatment of systemic sclerosis. Ann Rheum Dis. 2017;76(8):1327-1339. doi:10.1136/annrheumdis-2016-209909

20. Farge D, Burt RK, Oliveira MC, et al. Cardiopulmonary assessment of patients with systemic sclerosis for hematopoietic stem cell transplantation: recommendations from the European Society for blood and marrow transplantation autoimmune diseases working party and collaborating partners. Bone Marrow Transplant. 2017;52 (11):1495-1503. doi:10.1038/bmt.2017.56

21. Snowden JA, Saccard R, Allez M, et al. Haematopoietic SCT in severe autoimmune diseases: updated guidelines of the European Group for blood and marrow transplantation. Bone Marrow Transplant. 2012;47(6):770-790. doi:10.1038/bmt.2011.185

22. Snowden JA, Sharrack B, Akil M, et al. Autologous haematopoietic stem cell transplantation (aHSCT) for severe resistant autoimmune and inflammatory diseases - a guide for the generalist. Clin Med. 2018;18(4):329-334. doi:10.7861/clinmedicine.18-4-329

23. McSweeney PA, Nash RA, Sullivan KM, et al. High-dose immunosuppressive therapy for severe systemic sclerosis: initial outcomes. Blood. 2002;100(5):1602-1610. doi:10.1182/blood.V100.5.1602. h81702001602 $1602 \quad 1610$

24. Henes CJ, Koetter I, Horger M, et al. Autologous stem cell transplantation with thiotepa-based conditioning in patients with systemic sclerosis and cardiac manifestations. Rheumatology. 2014;53 (5):919-922. doi:10.1093/rheumatology/ket464

25. Burt RK, Farge D. Autologous HSCT is efficacious, but can we make it safer? Nat Rev Rheumatol. 2018;14(4):189-191. doi:10.1038/ nrrheum.2018.34

26. Martínez C, Urbano-Ispizua A, Rozman C, et al. Immune reconstitution following allogeneic peripheral blood progenitor cell transplantation: comparison of recipients of positive CD34+ selected grafts with recipients of unmanipulated grafts. Exp Hematol. 1999;27 (3):561-568. doi:10.1016/S0301-472X(98)00029-0

27. Kohno K, Nagafuji K, Tsukamoto H, et al. Infectious complications in patients receiving autologous CD34-selected hematopoietic stem cell transplantation for severe autoimmune diseases. Transpl Infect Dis. 2009;11(4):318-323. doi:10.1111/j.1399-3062.2009.00401.x

28. Oliveira MC, Labopin M, Henes J, et al. Does ex vivo CD34+ positive selection influence outcome after autologous hematopoietic stem cell transplantation in systemic sclerosis patients? Bone Marrow Transplant. 2016;51(4):501-505. doi:10.1038/bmt.2015.299

29. Ayano M, Tsukamoto H, Mitoma H, et al. CD34-selected versus unmanipulated autologous haematopoietic stem cell transplantation in the treatment of severe systemic sclerosis: a post hoc analysis of a phase I/II clinical trial conducted in Japan. Arthritis Res Ther. 2019;21(1):30. doi:10.1186/s13075-019-1823-0

30. Henes J, Oliveira MC, Lapobin M, et al. Autologous stem cell transplantation for progressive systemic sclerosis: a prospective non-interventional study from the European Society for blood and marrow transplantation autoimmune disease working party. Haematologica. 2021;106 (2):375-383. doi:10.3324/haematol.2019.230128

31. Henes JC, Schmalzing M, Vogel W, et al. Optimization of autologous stem cell transplantation for systemic sclerosis- a single-center longterm experience in 26 patients with severe organ manifestations. $J$ Rheumatol. 2012;39(2):269-275. doi: $10.3899 /$ jrheum. 110868
32. Burt RK, Oliveira MC, Shah S, et al. Cardiac involvement and treatment-related mortality after non-myeloablative haemopoietic stem-cell transplantation with unselected autologous peripheral blood for patients with systemic sclerosis: a retrospective analysis. Lancet. 2013;381(9872):1116-1124. doi:10.1016/S0140-6736(12)62114-X

33. Helbig G, Widuchowska M, Koglega A, et al. Safety profile of autologous hematopoietic stem cell mobilization and transplantation in patients with systemic sclerosis. Clin Rheumatol. 2018;37 (6):1709-1714. doi:10.1007/s10067-017-3954-5

34. Nakamura H, Odani T, Yasuda S, et al. Autologous haematopoietic stem cell transplantation for Japanese patients with systemic sclerosis: long-term follow-up on a Phase II trial and treatment- related fatal cardiomyopathy. Mod Rheumatol. 2018;28(5):879-884. doi:10.1080/14397595.2017.1416920

35. Guillaine-Jugnot P, Badoglio M, Labopin M, et al. Autologous haematopoietic stem cell transplantation (AHSCT) in autoimmune disease adult patients in France: analysis of the long-term outcome from the French Society for Bone Marrow Transplantation and Cellular Therapy (SFGM-TC). Clin Rheumatol. 2019;38(5):1501-1511. doi:10.1007/s10067-019-04435-2

36. van Bijnen S, Vries-Bouwstra J, van den Ende $\mathrm{CH}$, et al. Predictive factors for treatment-related mortality and major adverse events after autologous haematopoietic stem cell transplantation for systemic sclerosis: results of a long-term follow-up multicentre study. Ann Rheum Dis. 2020;79(8):1084-1089. doi:10.1136/annrheumdis-2020-217058

37. Henrique-Neto A, Vanconcelos MYK, Dias JBE, et al. Hematopoietic stem cell transplantation for systemic sclerosis: Brazilian experience. Adv Rheumatol. 2021;61(1):9. doi:10.1186/s42358-021-00166-8

38. Del Papa N, Onida F, Zaccara E, et al. Autologous hematopoietic stem cell transplantation has better outcomes than conventional therapies in patients with rapidly progressive systemic sclerosis. Bone Marrow Transplant. 2017;52(1):53-58. doi:10.1038/bmt.2016.211

39. Burt RK, Oliveira MC, Shah SJ. Cardiac assessment before stem cell transplantation for systemic sclerosis. JAMA. 2014;312(17):1803. doi:10.1001/jama.2014.12566

40. Burt RK, Shah SJ, Gheorghiade M, Ruderman E, Schroeder J. Hematopoietic stem cell transplantation for systemic sclerosis: if you are confused, remember: "it is a matter of the heart". J Rheumatol. 2012;39(2):206-209. doi:10.3899/jrheum.111302

41. Hosing C, Nash R, McSweeney P, et al. Acute kidney injury in patients with systemic sclerosis participating in hematopoietic cell transplantation trials in the United States. Biol Blood Marrow Transplant. 2011;17(5):674-681. doi:10.1016/j.bbmt.2010.08.003

42. Craciunescu OI, Steffey BA, Kelsey CR, et al. Renal shielding and dosimetry for patients with severe systemic sclerosis receiving immunoablation with total body irradiation in the scleroderma: cyclophosphamide or transplantation trial. Int J Radiat Oncol Biol Phys. 2011;79(4):1248-1255. doi:10.1016/j.ijrobp.2010.05.036

43. Dhesi S, Chu MP, Blevings G, et al. Cyclophosphamide- induced cardiomyopathy: a case report, review and recommendations for management. J Investig Med High Impact Case Rep. 2013;1(1):2324709613480346.

44. Allanore Y, Meune C, Vonk MC, et al. Prevalence and factors associated with left ventricular dysfunction in the EULAR Scleroderma Trial and Research group (EUSTAR) database of patients with systemic sclerosis. Ann Rheum Dis. 2010;69 (1):218-221. doi:10.1136/ard.2008.103382

45. Sandmeier B, Jäger VK, Nagy G, et al. Autopsy versus clinical findings in patients with systemic sclerosis in a case series from patients of the EUSTAR database. Clin Exp Rheumatol. 2015;33(4 Suppl 91):S75-S79.

46. Burt RK, Han X, Quigley K, et al. Cardiac safe hematopoietic stem cell transplantation for systemic sclerosis with poor cardiac function: a pilot safety study that decreases neutropenic interval to 5 days. Bone Marrow Transplant. 2021;56(1):50-59. doi:10.1038/s41409020-0978-2 
47. Snowden JA, Badoglio M, Labopin M, et al.; for European Society for Blood and Marrow Transplantation (EBMT) Autoimmune Diseases Working Party (ADWP); EBMT Paediatric Working Party (PWP); Joint Accreditation Committee of the International Society for Cellular Therapy (ISCT); EBMT (JACIE). Evolution, trends, outcomes, and economics of hematopoietic stem cell transplantation in severe autoimmune diseases. Blood Adv. 2017;1(27):2742-2755. doi:10.1182/bloodadvances.2017010041.

48. Farge D, Lapobin M, Tyndall A, et al. Autologous hematopoietic stem cell transplantation for autoimmune diseases: an observational study on 12 years' experience from the European Group for Blood and Marrow Transplantation Working Party on Autoimmune Diseases. Haematologica. 2010;95(2):284-292. doi:10.3324/ haematol.2009.013458

49. Denton CP, Khanna D. Systemic sclerosis. Lancet. 2017;390 (10103):1685-1699. doi:10.1016/S0140-6736(17)30933-9

50. Spierings J, van Rhenen A, Welsing PM, et al. A randomised, open-label trial to assess the optimal treatment strategy in early diffuse cutaneous systemic sclerosis: the UPSIDE study protocol. BMJ Open. 2021;11(3):e044483. doi:10.1136/bmjopen-2020-044483

51. Ait Abdallah N, Wang M, Lansiaux P, et al. Long term outcomes of the French ASTIS systemic sclerosis cohort using the global rank composite score. Bone Marrow Transplant. 2021;56(9):2259-2267. doi:10.1038/s41409-021-01355-1

52. Clements PJ, Hurwitz EL, Wong WK, et al. Skin thickness score as a predictor and correlate of outcome in systemic sclerosis: high-dose versus low-dose penicillamine trial. Arthritis Rheum. 2000;43 (11):2445-2454. doi:10.1002/1529-0131(200011)43:11<2445::AIDANR11>3.0.CO;2-Q

53. Costa-Pereira KR, Guimaraes AL, Moraes DA, et al. Hematopoietic stem cell transplantation improves functional outcomes of systemic sclerosis patients. J Clin Rheumatol. 2020;26(7SSuppl 2):S131S138. doi:10.1097/RHU.0000000000001117

54. Khanna D, Furst DE, Clements PJ, et al. Standardization of the modified Rodnan skin score for use in clinical trials of systemic sclerosis. $J$ Scleroderma Relat Disord. 2017;2(1):11-18. doi: $10.5301 /$ jsrd.5000231

55. Daikeler T, Kump E, Stern M, et al. Autologous hematopoietic stem cell transplantation reverses skin fibrosis but does not change skin vessel density in patients with systemic sclerosis. Pathol Biol. 2015;63(4-5):164-168. doi:10.1016/j.patbio.2015.07.006

56. Wada DT, Almeida FA, Moraes DA, et al. Automatic quantitative computed tomography evaluation of the lungs in patients with systemic sclerosis treated with autologous stem cell transplantation. J Clin Rheumatol. 2020;26(7s Suppl 2):S158-S164. doi:10.1097/ RHU.0000000000001242

57. Kloth C, Thaiss WM, Preibsch H, et al. Quantitative chest CT analysis in patients with systemic sclerosis before and after autologous stem cell transplantation: comparison of results with those of pulmonary function tests and clinical tests. Rheumatology. 2016;55 (10):1763-1770. doi:10.1093/rheumatology/kew259

58. Ciaff J, van Leeuwen NM, Boonstra M, et al. Evolution of interstitial lung disease one year after hematopoietic stem cell transplantation or cyclophosphamide for systemic sclerosis. Arthritis Care Res. 2020. doi:10.1002/acr.24451

59. Maltez N, Puyade M, Wang M, et al. Association of autologous hematopoietic stem cell transplantation in systemic sclerosis with marked improvement in health-related quality of life. Arthritis Rheumatol. 2021;73(2):305-314. doi:10.1002/art.41519

60. Puyade M, Maltez N, Lansiaux P, et al. Health-related quality of life in systemic sclerosis before and after autologous haematopoietic stem cell transplant- a systematic review. Rheumatology. 2020;59 (4):779-789. doi:10.1093/rheumatology/kez300
61. Steen VD, Medsger TA Jr. Fertility and pregnancy outcomes in women with systemic sclerosis. Arthritis Rheum. 1999;42 (4):763-768. doi:10.1002/1529-0131(199904)42:4<763::AIDANR21>3.0.CO;2-V

62. Taborelli M, Ramoni V, Brucato A, et al. Brief report: successful pregnancies but a higher risk of preterm births in patients with systemic sclerosis: an Italian multicenter study. Arthritis Rheum. 2012;64(6):1970-1977. doi:10.1002/art.34350

63. Harward LE, Mitchell K, Pieper C, Copland S, Criscione-Schreiber LG, Clowse ME. The impact of cyclophosphamide on menstruation and pregnancy in women with rheumatologic disease. Lupus. 2013;22 (1):81-86. doi:10.1177/0961203312468624

64. Nowlin NS, Brick JE, Weaver DJ, et al. Impotence in scleroderma. Ann Intern Med. 1986;104(6):794-798. doi:10.7326/0003-4819-104-6-794

65. Krittian SM, Saur SJ, Schloegl A, et al. Erectile function and connective tissue diseases. Prevalence of erectile dysfunction in German men with systemic sclerosis compared to other connective tissue diseases and healthy subjects. Clin Exp Rheumatol. 2021;39(Suppl 131):52-56.

66. Snarski E, Snowden JA, Oliveira MC, et al. Onset and outcome of pregnancy after autologous haematopoietic SCT (AHSCT) for autoimmune disease: a retrospective study of the EBMT aUtoimmune Diseases Working Party (ADWP). Bone Marrow Transplant. 2015;50 (2):216-220. doi:10.1038/bmt.2014.248

67. Massenkeil G, Alexander T, Rosen O, et al. Long-term follow-up of fertility and pregnancy in autoimmune diseases after autologous haematopoietic stem cell transplantation. Rheumatol Int. 2016;36 (11):1563-1568. doi:10.1007/s00296-016-3531-2

68. Somers EC, Thomas SL, Smeeth L, Hall AJ. Are individuals with an autoimmune disease at higher risk of a second autoimmune disorder? Am J Epidemiol. 2009;169(6):749-755. doi:10.1093/aje/kwn408

69. Daikler T, Labopin M, Di Gioia M, et al. Secondary autoimmune disease occurring after HSCT for an autoimmune disease: a retrospective study of the EBMT autoimmune disease working party. Blood. 2011;118 (6):1693-1698. doi:10.1182/blood-2011-02-336156

70. Onishi A, Sugiyama D, Kumagai S, Morinobu A. Cancer incidence in systemic sclerosis: meta-analysis of population-based cohort studies. Arthritis Rheum. 2013;65(7):1913-1921. doi:10.1002/art.37969

71. Wooten M. Systemic sclerosis and malignancy: a review of the literature. South Med J. 2008;101(1):59-62. doi:10.1097/ SMJ.0b013e31815838ce

72. Franks JM, Martyanov V, Wang Y, et al. Machine learning predicts stem cell transplant response in severe scleroderma. Ann Rheum Dis. 2020;79(12):1608-1615. doi:10.1136/annrheumdis-2020-217033

73. Showalter K, Spiera R, Magro C, et al. Machine learning integration of scleroderma histology and gene expression identifies fibroblast polarization as a hallmark of clinical severity and improvement. Ann Rheum Dis. 2021;80(2):228-237. doi:10.1136/annrheumdis-2020-217840

74. EBMT ADWP prospective non-interventional Study: post-AHSCT management in SSC patients (NISSC-2). Available from: https:// clinicaltrials.gov/ct2/show/NCT03444805. NLM identifier: NCT03444805. Accessed September 6, 2021.

75. Scleroderma Treatment With Autologous Transplant (STAT) Study. Available from: https:/clinicaltrials.gov/ct2/show/NCT01413100. NLM identifier: NCT01413100. Accessed September 6, 2021.

76. Tsuchikama K, An Z. Antibody-drug conjugates: recent advances in conjugation and linker chemistries. Protein Cell. 2018;9(1):33-46. doi: 10.1007/s13238-016-0323-0

77. Palchaudhuri R, Saez B, Hoggatt J, et al. Non-genotoxic conditioning for hematopoietic stem cell transplantation using a hematopoietic-cell-specific internalizing immunotoxin. Nat Biotechnol. 2016;34(7):738-745. doi:10.1038/nbt.3584 


\section{Publish your work in this journal}

The Journal of Blood Medicine is an international, peer-reviewed, open access, online journal publishing laboratory, experimental and clinical aspects of all aspect pertaining to blood based medicine including but not limited to: Transfusion Medicine; Blood collection, Donor issues, Transmittable diseases, and Blood banking logistics; Immunohematology; Artificial and alternative blood based therapeutics; Hematology; Biotechnology/nanotechnology of blood related medicine; Legal aspects of blood medicine; Historical perspectives. The manuscript management system is completely online and includes a very quick and fair peer-review system. Visit http://www.dovepress.com/testimonials.php to read real quotes from published authors. 